\title{
L'anatomie andrologique dans les écrits de Jules Germain Cloquet (1790-1883), grand chirurgien et anatomiste
}

\author{
Georges ANDROUTSOS
}

Histoire de la Médecine, Faculté de Médecine, Université d'Ioannina, Grèce

\begin{abstract}
RESUME
Cloquet fut un grand anatomiste et chirurgien. Dans ses écrits qui englobent divers domaines tels que l'anatomie et la physiologie normales, l'anatomie pathologique, l'histoire naturelle, la pathologie et la chirurgie, l'anatomie andrologique tient une place considérable et met en évidence le haut niveau des connaissances de Cloquet en matière d'andrologie.
\end{abstract}

Mots clés : Cloquet, chirurgien, anatomiste, andrologie

Cloquet (Figure 1) naquit le 28 décembre 1790 à Paris. Son père, Jean Baptiste Antoine (décédé en 1828), fut dessinateur et graveur. Sa mère était Claude Louise Lajude. Il épousa en premières noces, Juliette Lebreton (décédée en 1842) dont il eut une fille. En secondes noces il épousa, le 19 septembre 1846, France Marie Corney. Il mourut le 23 février 1883 à Paris.

\section{ETUDES-CARRIERE}

Cloquet avait à peine seize ans (1806) lorsqu'il fut nommé par le ministre de l'intérieur élève de l'école d'anatomie artificielle établie à Rouen. II y obtint en

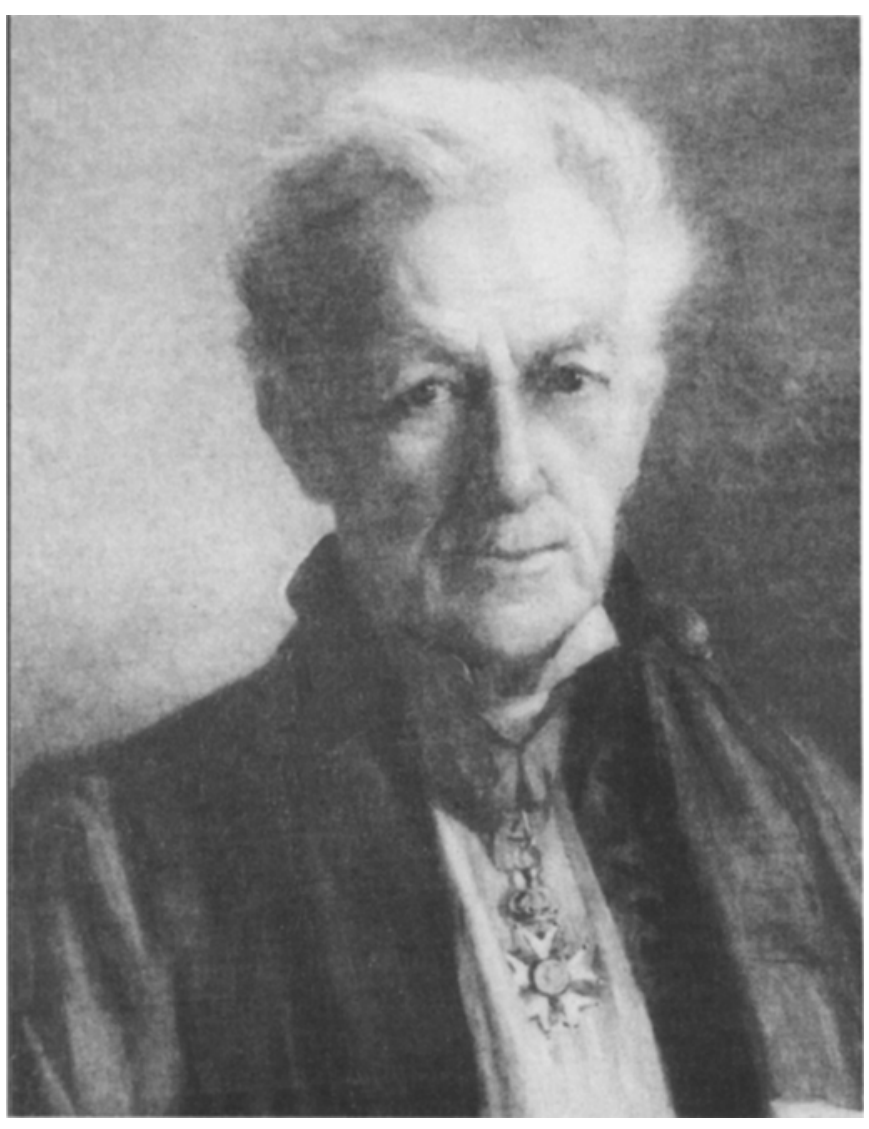

Figure 1 : Cloquet revêtu de la robe professorale. Ce tableau se trouve dans l'Académie nationale de Médecine

Correspondance :

Dr Georges ANDROUTSOS - 1 rue Ipeirou, 10433, Athènes, Grèce - Email lyon 48@otenet.gr 
1809 le prix de botanique. En 1810, il fit des cours particuliers d'anatomie générale, de pathologie chirurgicale et d'opérations pour gagner sa vie. En 1811, il obtint, au concours, une place d'interne des hôpitaux de Paris et fut préparateur d'anatomie artificielle. Dispensé du service militaire en 1812, sur la demande des professeurs de l'Ecole de médecine, il partageait l'année suivante, avec Pierre Rayer (1793-1867), le premier prix d'anatomie et de physiologie de l'Ecole pratique ; à partir de ce moment, il n'est pas de concours ouvert à la faculté dans lequel il n'ait paru avec avantage [2].

A cette époque d'émulation et de travail où les moindres positions étaient ardemment disputées, il n'était pas facile de se frayer sa voie au milieu de tant d'hommes de valeur ; mais le jeune Cloquet unissait à une intelligence hors ligne une force de volonté que rien ne pouvait abattre et une puissance de travail qui triomphe de tous les obstacles. Toujours sur la brèche, doué d'une activité infatigable, il multipliait et variait ses recherches, et pendant cette période de sa vie, il n'est guère d'année où il n'ait produit quelque étude intéressante.

Après avoir emporté de haute lutte la place de prosecteur de la faculté en 1815, il disputa à Gilbert Breschet (17851843 ), en 1819, celle de chef des travaux anatomiques, laissée vacante par la nomination de Pierre Béclard (1785-1825) au grade de professeur, et Breschet ne l'emporta que grâce à l'habileté manuelle du grand préparateur Jean Bogros (1786-1825), qui lui prépara ses pièces. Cette même année, Cloquet fut nommé au concours chirurgien en chef adjoint de l'hôpital SaintLouis, et deux ans après, étant à peine âgé de trente et un ans, il entrait à l'Académie de médecine. Lors du premier concours ouvert pour l'agrégation, en exécution de l'arrêté du 12 avril 1823, Cloquet obtint la première des quatre places qui furent données dans la section de chirurgie. En 1825, il fut porté en troisième ligne sur la liste de propositions dressée par la faculté pour la chaire d'anatomie de Béclard [La liste se composait de Jean Cruveilhier (1791-1774), Gilbert Breschet et Cloquet. Cruveilhier fut nommé le 21 mai 1825]. Enfin, quand le concours eut été rétabli pour le professorat, il descendit une dernière fois dans cette arène où il avait conquis tous ses titres, et fut nommé, le 24 mars 1831 , professeur de pathologie externe à la presque unanimité des suffrages [ll avait pour compétiteurs : Louis Joseph Sanson (1790-1841), Alfred Velpeau (1790-1867), Frédéric Blandin (1798-1849), Auguste Bérard (18021846), Pierre Gerdy (1797-1856), Thierry et Alexis Petit]. Deux ans après, il quitta cette chaire pour celle de clinique externe qu'Antoine Dubois (1795-1871) venait d'abandonner et qu'il a occupée jusqu'en 1850, époque à laquelle il revint à son premier enseignement. En 1851, Cloquet devint chirurgien de l'empereur Napoléon III. II lui manquait un dernier titre. L'Académie des sciences, qui avait accueilli ses premiers travaux avec tant de faveur et qui lui avait dans sa jeunesse décerné deux couronnes (la première, en 1818, pour son Mémoire sur les calculs urinaires), ne lui avait pas encore ouvert ses portes. Cette omission fut réparée en 1855 , et Cloquet vint occuper la place où s'était assis André Marie Lallemand (1750-1834). En 1858 il devint professeur honoraire et conserva le droit d'assistance et de délibération dans les assemblées générales de la faculté ainsi que la possibilité de faire partie du jury institué pour les concours d'agrégation [3].

II fut membre de nombreuses académies étrangères. II est fait baron en 1867. Commandeur de la Légion d'honneur. Lauréat de l'Institut pour le prix proposé par l'Académie des sciences en 1818 et 1822 . Médaille du gouvernement pour les soins apportés aux cholériques (1831).

\section{SON CEUVRE}

En dépit du travail incessant que nécessitaient ces luttes répétées, malgré les soins de son enseignement et de sa riche clientèle, Cloquet n'a jamais cessé de se livrer à son penchant pour les recherches scientifiques, et bien qu'il n'ait produit, en fait d'ouvrages de longue haleine, que son bel atlas sur l'anatomie de l'homme, qui lui demanda près de vingt ans de travail, et qui est resté comme un modèle d'exactitude et de perfection iconographique, il est peu de chirurgiens qui aient autant écrit et qui aient abordé autant de sujets différents. L'anatomie et la physiologie normales, l'anatomie pathologique, l'histoire naturelle, la pathologie, la clinique chirurgicale, la médecine opératoire, ont été tour à tour l'objet de ses travaux. Dans l'impossibilité de les énumérer tous, nous nous bornerons à signaler ceux qui ont eu le plus de retentissement [La liste des publications scientifiques de Cloquet, depuis 1818 jusqu'à 1854, a été dressée par lui même. Elle ne comprend pas moins de quatre-vingts articles différents et forme une brochure in $-4^{\circ}$ de 72 p., sous ce titre : Notice analytique des travaux de $M$. Jules Cloquet à l'appui de sa candidature à l'Académie des sciences, section de médecine et de chirurgie, Paris, 1854].

A ce titre nous citerons d'abord, en anatomie, ses trois mémoires sur l'appareil de la vision, ses recherches sur le crémaster, les ligaments ronds, les fibrocartilages ; en histoire naturelle, ses mémoires sur les vers intestinaux, et sur les voies lacrymales des serpents ; en anatomie pathologique, ses travaux sur les hernies, sur le phénomène de l'effort ; en médecine opératoire, 
ses procédés pour les amputations, pour le bec-delièvre, pour l'ablation des loupes, pour la ligature de la langue, sa thèse d'agrégation sur l'extraction de la cataracte, sa méthode de cautérisation pour l'oblitération des ouvertures fistuleuses. II étudia aussi le traitement dans la rupture du périnée. Nous passons sous silence les nombreux articles qu'il a insérés dans le Dictionnaire de médecine en vingt et un volumes et les instruments dont il a doté la chirurgie. Un certain nombre d'entre eux, tels que sa sonde à double courant, sa pince porteligature et sa pince œsophagienne, sont restés pendant longtemps dans la pratique; quelques autres, comme son sécateur pour l'excision des amygdales, ont servi de point de départ à des perfectionnements utiles. II étudia le canal péritonéo-vaginal et donna la description du " ganglion de Cloquet ». If donna la première description du canal vitreux (canal hyaloïde, 1821).

En 1830, il réalisa la première opération sous anesthésie par hypnose, une mammectomie. Passionné par l'acupuncture, il expérimenta l'analgésie par acupuncture et publia, avec le docteur Dantu, un Traité de l'acupuncture, étudiée surtout dans le traitement des syndromes douloureux. II devint le confident de Gustave Flaubert, qu'il accompagna en Corse et soigna pour son épilepsie.

Il a enrichi le muséum de la faculté de plusieurs pièces anatomopathologiques.

\section{SES CEUVRES MAIITRESSES}

Thèse de doctorat: Recherches sur les hernies de l'abdomen. Paris, 1817. Atlas d'anatomie de l'homme. Paris, 1821. Traité de l'acupuncture. Paris,1821. Thèse d'agrégation : An curanda oculi suffusione (vulgo cataracte) lentis crystallinae extractio hujus depressione praestantior. Didot, Paris, 1824. Manuel d'anatomie descriptive. Didot, Paris, 1825.

Traité d'anatomie. Paris, 1830, 5 vol. Pathologie chirurgicale. Plan et méthode qu'il convient de suivre dans l'enseignement de cette science. Fournier, Paris 1831, 151 p. Souvenir sur la vie privée du général Lafayette. Galignani, Paris, 1836, 394p. Anatomie de l'homme ou description et figures lithographiées de toutes les parties du corps humain. Lasteyrie, Paris, 1821-1831, 5 tomes de texte en 3 vol. et 3 vol. de planches.

\section{DISCUSSION}

Presque tous ses travaux appartiennent à la période ascendante de sa carrière et sont antérieurs à son arrivée au professorat. A partir du jour où il parvint à cette position, conquise au prix de tant d'efforts, Cloquet sentit son ambition satisfaite. Le désir de se placer au premier rang ne le porta jamais à y sacrifier le bonheur de sa vie. La profondeur et l'étendue de ses connaissances, son talent comme professeur, la sympathie qu'excitaient autour de lui son aménité, son tect exquis et la distinction de ses manières, auraient pu lui permettre de se poser en chef d'école ; mais ses goûts simples et modestes, son amour pour l'étude, un certain éloignement pour le côté pratique de la chirurgie le préservèrent de ce danger. La longue carrière de Cloquet n'est pas seulement un témoignage éclatant de ce que peuvent faire l'intelligence et le travail réunis, elle est un exemple rare de la somme de bonheur que peut comporter une existence médicale bien remplie, lorsqu'on a le ccur assez noble et la raison assez haute pour le chercher à sa véritable source : l'estime et l'affection de ses confrères et les joies tranquilles de l'étude [4].

\section{L'ANATOMIE ANDROLOGIQUE DANS SON OEUVRE}

Nous empruntons les passages suivants du Manuel de l'anatomie déscriptive... [1].

Les organes génitaux de l'homme qui sont très nombreux, doivent être décrits dans l'ordre physiologique de leurs fonctions. Ce sont : 1) les testicules et leurs enveloppes ; 2) les canaux déférents et le cordon testiculaire ; 3 ) les vésicules spermatiques et les canaux éjaculateurs ; 4) le pénis et les organes qui en dépendent.

\section{Des testicules et de leurs enveloppes}

Les testicules sont deux organes glanduleux logés dans les bourses, et destinés à sécréter le sperme. Ils sont enveloppés par des tuniques dont il est avantageux de faire d'abord la description. L'une de ces tuniques, ou la plus extérieure, est commune aux deux testicules : c'est le scrotum ; les autres, qui sont au nombre de cinq, sont particulières à chacun de ces organes, et placées, de dehors en dedans, dans l'ordre suivant : 1) le dartos ; 2) la tunique érythroïde ou le muscle crémaster ; 3) la tunique commune au cordon et au testicule, ou la tunique fibreuse ; 4) la tunique vaginale ; 5) la tunique albuginée.

Le scrotum est l'enveloppe cutanée des testicules. Ce n'est pas autre chose qu'un prolongement de la peau de la partie interne des cuisses, du périnée et de la verge. Cette membrane est remarquable par sa couleur brune, par de nombreuses rugosités qui la sillonnent, surtout lorsque les bourses sont contractées sur ellesmêmes ; par la grande quantité de follicules sébacés qu'elle contient, et par les poils longs et peu abondants qui s'y développent chez l'adulte. Une ligne médiane, 
rugueuse, saillante, nommée le raphé, et qui se prolonge depuis la partie antérieure de l'anus jusqu'à la racine de la verge, partage le scrotum en deux moitiés. Le scrotum a la même organisation que la peau en général ; seulement son chorion est très mince, et laisse apercevoir par sa transparence les vaisseaux sanguins qui rampent dans le dartos.

On appelle dartos la seconde enveloppe des testicules, que les anciens anatomistes croyaient de nature musculaire. Cette tunique est formée chez l'adulte par une expansion fibrocelluleuse de l'aponévrose superficielle de l'abdomen (fascia superficialis), qui abandonne l'anneau inguinal pour se jeter sur le cordon testiculaire qu'elle renferme, ainsi que le testicule et la tunique vaginale. Elle paraît contractile, et est considérée par Meckel comme formant le passage du tissu cellulaire au tissu musculaire ; d'autres anatomistes en font un tissu particulier, sous le nom de dartoïde. Par sa face externe, elle est unie à la peau du scrotum, et s'adosse en dedans avec celle du côté opposé pour former la cloison des bourses ; elle s'attache aussi dans ce sens à la branche de l'ischion, et passe sur les côtés du pénis pour se continuer avec son ligament sus-penseur. Du fond de l'espèce de poche que forme le dartos s'élève un cordon fibrocelluleux, triangulaire, blanchâtre, creux, lequel se fixe à la partie du testicule et de l'épididyme qui n'est point recouverte par la tunique vaginale. Cette expansion est produite par l'extrémité supérieure du gubernaculum testis, dont la base s'est épanouie, en se renversant, pour recevoir le testicule lors de sa sortie de l'abdomen chez le fœtus, et lui former une espèce de sac qui n'est que le dartos luimême.

La membrane érythroïde n'est autre que le muscle crémaster. La membrane fibreuse ou la tunique commune au cordon et au testicule est un prolongement infundibuliforme de l'aponévrose transverse (fascia transversalis) ; la gaine qu'il forme autour du cordon est un long tuyau, facile à isoler, qui descend avec lui en parcourant le canal inguinal, et l'accompagne jusqu'au bord supérieur du testicule. Là il se perd dans le tissu cellulaire extérieur de la tunique vaginale.

La tunique vaginale (Figure 2) est la membrane séreuse qui enveloppe le testicule. Elle forme un sac sans ouverture qui se réfléchit sur cet organe et sur l'épididyme qu'elle recouvre, sans cependant les contenir dans sa cavité. Sa face interne est lisse, polie, lubrifiée par de la sérosité ; sa face externe adhère d'une part à la tunique fibreuse, et de l'autre elle recouvre le testicule et l'épididyme. Avant la descente du testicule dans les bourses, la tunique vaginale n'existe pas. Elle est formée par un prolongement que le péritoine fournit à cet organe lorsqu'il sort de l'abdomen, et qui se rétrécit ensuite, s'en sépare et s'en isole entièrement pour former une membrane distincte.

Les testicules (Figure 3) sont deux organes éminemment vasculaires, situés à la partie inférieure des cordons testiculaires, auxquels ils semblent pour ainsi dire suspendus : celui du côté droit est un peu plus haut que celui du côté gauche. Leur consistance, assez grande chez les adultes, diminue beaucoup dans la vieillesse, de même que leur volume. Leur forme est celle d'un ovoïde comprimé de droite à gauche ; leur direction et leur diamètre sont un peu obliques, en sorte que l'on peut $y$ distinguer deux faces latérales légèrement convexes, un bord inférieur incliné en devant, un bord supérieur tourné en arrière, lequel est côtoyé par un corps appelé l'épididyme, et deux extrémités, dont l'une est antérieure et supérieure, l'autre postérieure et inférieure. Les testicules (Figure 4) sont renfermés immédiatement dans une coque fibreuse que leur forme la tunique albuginée, laquelle est une membrane fibreuse, d'un blanc opaque, d'un tissu serré, forte, résistante, et pourtant extensible et rétractile tout à la fois. En dehors, elle est recouverte par la tunique vaginale ; en dedans, elle envoie un grand nombre de prolongements aplatis, filiformes, qui produisent des cloisons incomplètes dans la cavité de l'espèce de coque qu'elle représente. Les loges triangulaires que séparent ces cloisons sont remplies par les vaisseaux séminifères. En haut, la tunique albuginée présente un renflement appelé corps de Highmore, à travers lequel passent les troncs de ces vaisseaux, qui vont se rendre à l'épididyme. Le parenchyme des testicules est très mou, et se présente sous l'apparence d'une pulpe jaunâtre ou grise renfermée dans les cloisons dont il vient d'être parlé. Cette sorte de pulpe est formée par une immense quantité de filaments très tenus, flexueux, entrelacés, repliés, et lâchement unis les uns aux autres par un tissu cellulaire très fin, lequel est parcouru par des ramifications capillaires sanguines. Alexander Monro estime leur nombre à soixante-deux mille cinq cents, tandis que des auteurs modernes ne le portent qu'à trois cents environ. Chacun d'eux a seize pieds de long sur un deux centième de pouce de large ; en sorte que, placés les uns à la suite des autres, ils représenteraient une longueur très considérable. Ces filaments sont les vaisseaux ou conduits séminifères : on n'a pas encore démontré leur cavité au moyen des injections. Ils présentent de distance en distance de petits renflements indiqués par certains anatomistes comme des granulations glanduleuses, et par d'autres comme de simples replis. Ils se dirigent tous vers le bord supérieur du testicule, et se réunissent, avant d'y parvenir, en quinze ou vingt troncs qu'on nomme afférents, et qui 


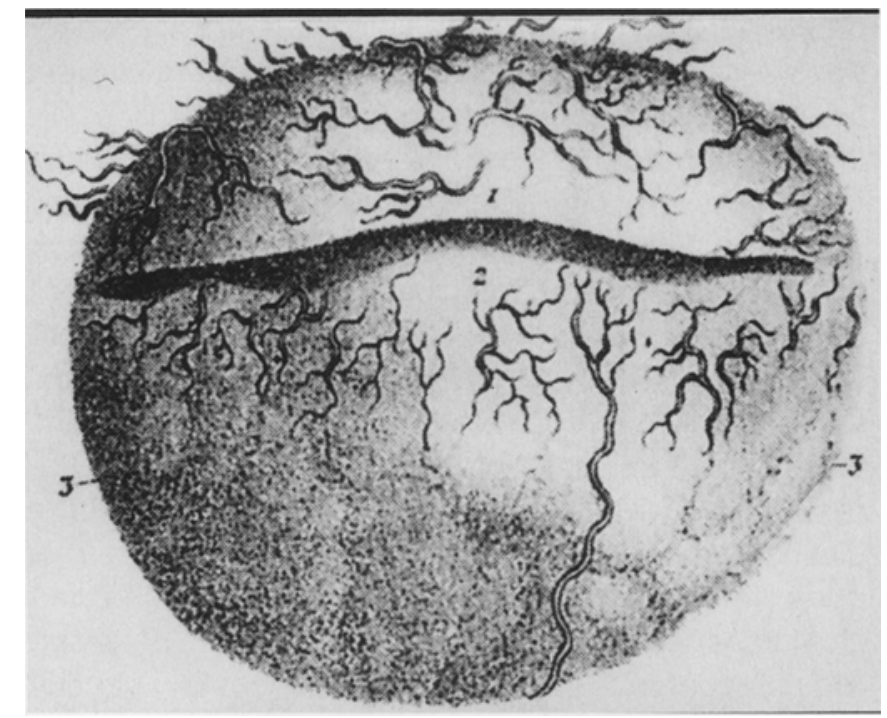

Figure 2 : La tunique vaginale.

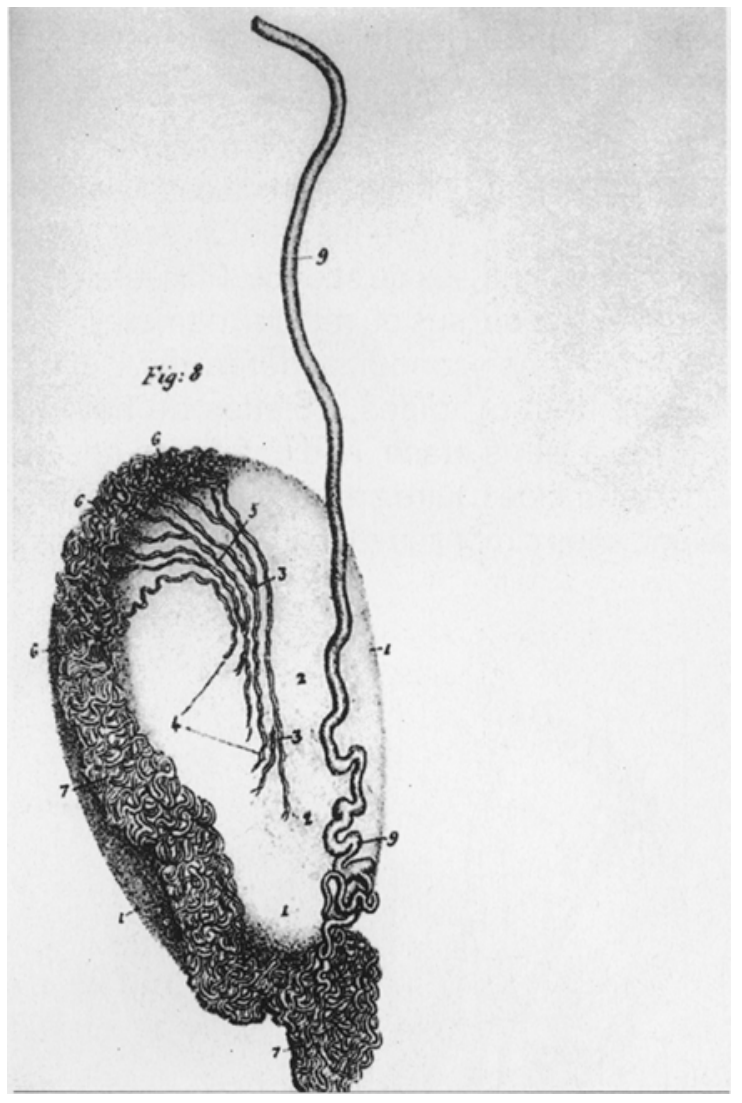

Figure 4 : Le canal déférent et l'épididyme.

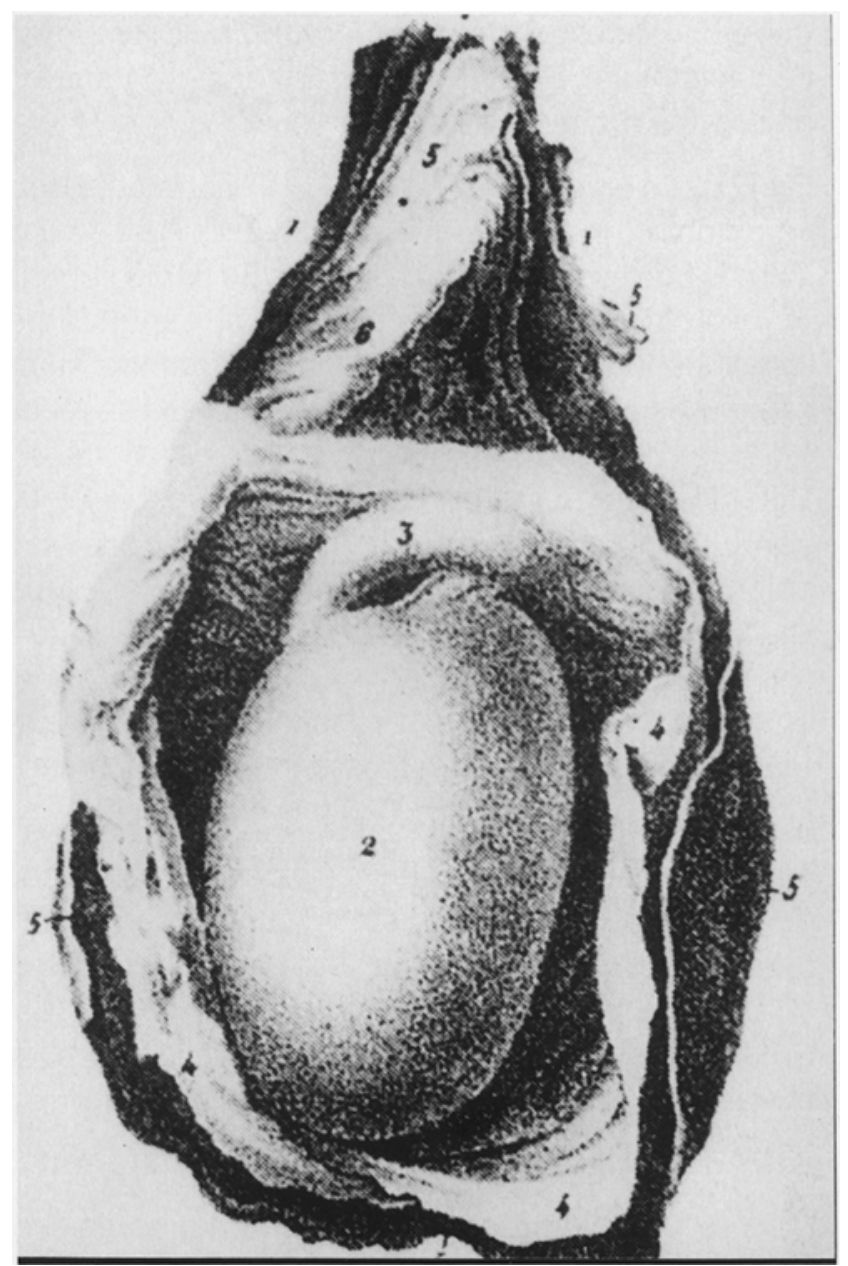

Figure 3 : Les testicules. 
traversent le corps de Highmore au-dessous de la tête de l'épididyme, dans lequel ils se rendent pour donner naissance au canal déférent.

On appelle épididyme (Figure 4), un petit corps oblong, vermiforme, renflé à ses extrémités, qui est couché le long du bord supérieur du testicule. Sa partie supérieure ou sa tête embrasse l'extrémité correspondante du testicule, dont elle reçoit les canaux afférents ; sa partie inférieure ou sa queue est plus rétrécie : elle se recourbe en haut, et se continue avec le canal déférent. L'épididyme n'est autre chose, d'après Meckel, qu'un conduit simple, grêle et flexueux, dans lequel s'abouchent tous ceux qui ont traversé le corps de Highmore ; son volume augmente à mesure qu'il se rapproche du canal déférent. Ses parois sont fort épaisses par rapport à son calibre. Sa longueur est considérable : Monro l'estime à trente-deux pieds.

Les tuniques du testicule reçoivent des branches des artères épigastriques, honteuses externes, et des artères de la cloison. Le testicule et l'épididyme reçoivent des branches des artères spermatiques ; leurs veines forment le plexus pampiniforme ; leurs vaisseaux lymphatiques vont se jeter dans les ganglions lombaires, et leurs nerfs viennent du plexus spermatique.

\section{Du cordon testiculaire et du canal déférent}

L'artère et la veine spermatiques, d'autres vaisseaux sanguins dont le volume est médiocre et l'existence variable, des vaisseaux lymphatiques, des nerfs venus du plexus spermatique et de la branche génito-crurale du plexus lombo-abdominal, et enfin le canal déférent, constituent, par leur réunion, le cordon des vaisseaux spermatiques ou testiculaires, dans lequel on trouve aussi, souvent, un prolongement fibreux et filiforme qui unit le péritoine à la tunique vaginale. Un tissu cellulaire abondant, continuation de celui qui, placé entre le péritoine et l'aponévrose du muscle transverse de l'abdomen, constitue le fascia propria, réunit toutes ces parties ; et le cordon qui résulte de leur ensemble, plus court ordinairement à droite qu'à gauche, monte presque verticalement vers le bord supérieur de la branche horizontale du pubis, se recourbe en dehors et en arrière pour pénétrer dans l'abdomen à travers le canal inguinal, en croisant la direction de l'artère épigastrique, placée au-dessous et en dedans de lui.

Le canal déférent (Figure 4), ou le canal excréteur du sperme, naît de la queue de l'épididyme, dont il n'est que la continuation, et remonte flexueux derrière le testicule : il s'engage dans le cordon des vaisseaux spermatiques, en arrière et en dedans de l'artère du même nom, franchit le canal inguinal, et, descendant en arrière et en dedans, il se porte derrière l'artère ombilicale, pour gagner la partie postérieure et inférieure de la vessie, en passant obliquement derrière l'uretère. Là il s'aplatit, se rapproche de celui du côté opposé, et il marche presque horizontalement le long du bord interne des vésicules séminales, en redevenant flexueux comme à son origine : arrivé à la base de la prostate, il reçoit le conduit des vésicules, et se continue avec le conduit éjaculateur. Le canal déférent augmente successivement de volume depuis son origine jusqu'à sa terminaison. Sa cavité est fort étroite relativement à ses parois, qui sont blanches, très épaisses, très résistantes, et composées de deux membranes distinctes. La membrane externe, dont l'épaisseur est quelquefois d'une demi ligne, paraît comme cartilagineuse. La membrane interne adhère assez peu à la précédente. Continue avec la membrane muqueuse de l'urètre, elle est lisse dans toute son étendue, excepté à l'endroit de son union avec la membrane correspondante des vésicules séminales, où elle est réticulée.

\section{Des vésicules séminales, de la prostate et des glandes de Cowper}

Les vésicules séminales ou spermatiques (Figure 5) paraissent n'être qu'une dilatation du canal déférent, plus ou moins repliée sur elle-même. Ce sont deux petits réservoirs membraneux du sperme. Placées au-dessous de la vessie, au-dessus du rectum, derrière la prostate, en dehors des conduits déférents, elles sont irrégulièrement conoïdes, aplaties de haut en bas, bosselées à leur surface, et d'une teinte grisâtre, très éloignées l'une de l'autre en arrière, et très rapprochées en avant, elles circonscrivent au-delà de la prostate un

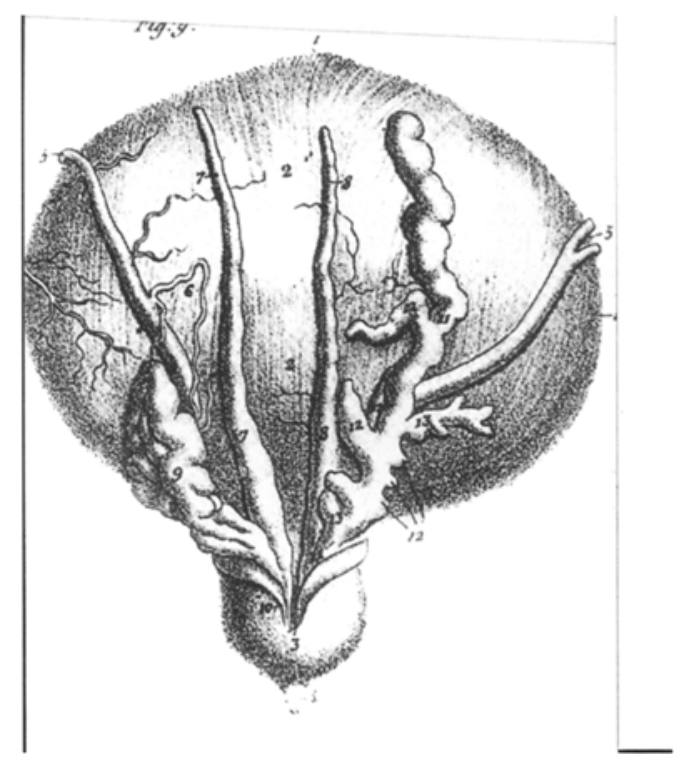

Figure 5 : Les vésicules séminales. 
espace triangulaire dans lequel la vessie est en rapport avec le rectum. Leur extrémité postérieure ou leur fond se termine par un cul-de-sac arrondi, assez large ; leur extrémité antérieure ou leur col est étroite, allongée, et se continue avec le conduit excréteur de cette vésicule, qui va se joindre au canal déférent, et forme avec lui de canal éjaculateur. L'intérieur des vésicules offre plusieurs cellules assez profondes, séparées par des demi cloisons, qui communiquent toutes ensemble. Ces cellules sont revêtues par une membrane muqueuse fort ténue, réticulée, et qui offre de l'analogie avec la membrane interne de la vésicule biliaire. Au-dessous d'elle on trouve une autre membrane assez dense, blanchâtre, fibrocelluleuse suivant la plupart des anatomistes, mais musculaire suivant Amussat, qui est parvenu à déplisser les vésicules spermatiques, et qui compare la structure de ces organes à celle de la vésicule du fiel.

On connaît sous le nom de conduits éjaculateurs (Figure 6), deux canaux formés par la jonction à angle droit des conduits déférents avec ceux qui terminent les vésicules séminales. Ils sont coniques, longs d'un pouce environ, et marchent obliquement de bas en haut et d'arrière en avant dans l'épaisseur de la prostate, s'accolent l'un à l'autre, et s'ouvrent dans l'urètre ordinairement par deux petits orifices oblongs, situés sur le sommet du verumontanum. Ils portent dans l'urètre le sperme qui sort des vésicules, et celui qui vient directement du testicule par le canal déférent.

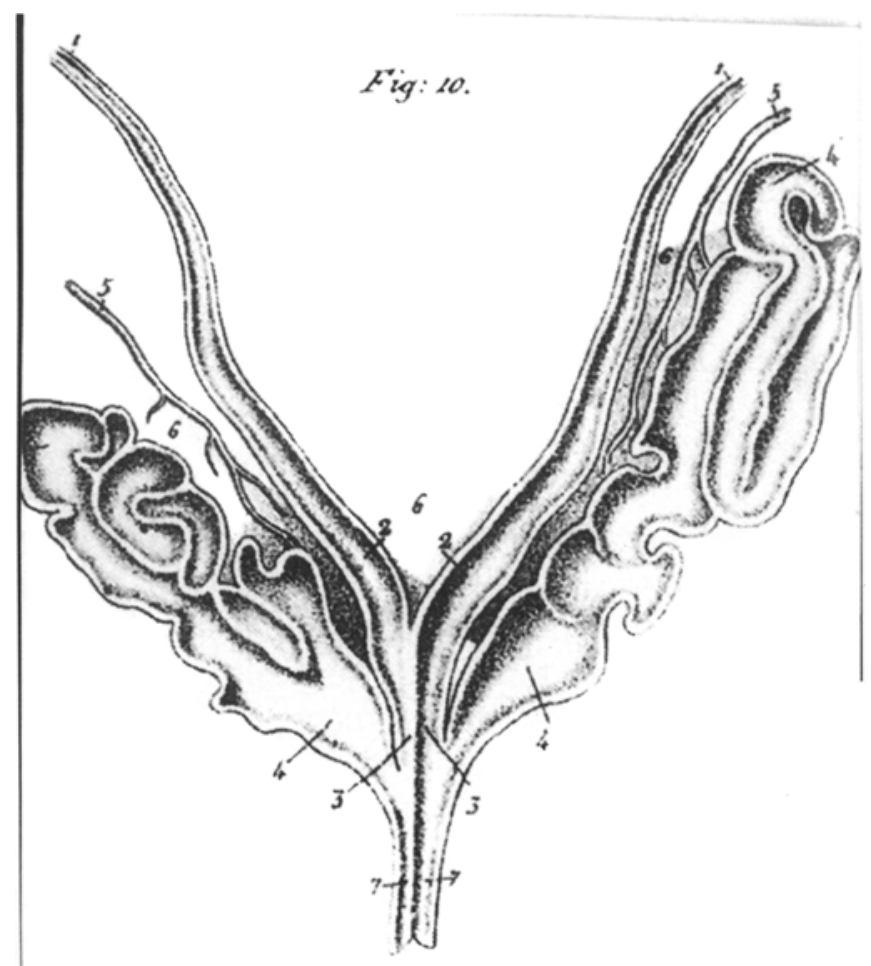

Figure 6 : Les conduits éjaculateurs.
Les vésicules séminales reçoivent leurs vaisseaux des artères hypogastriques, et leurs nerfs du plexus du même nom.

La prostate (Figure 7) est un organe folliculeux, situé entre le rectum et la symphyse pubienne, au-devant du col de la vessie, et embrassant l'origine de l'urètre. Sa direction change suivant que le rectum est plein ou vide. Son volume augmente en général avec l'âge, depuis l'enfance jusqu'à la vieillesse, et dans sa plus grande largeur il a deux lignes de moins chez l'adulte de dix-huit à vingt-cinq ans, que chez l'homme de quarante ans : il présente d'ailleurs de telles différences, suivant les individus, qu'il est presque impossible d'en déterminer rigoureusement les diamètres, ainsi qu'on avait essayé de le faire.

La prostate a la forme d'un cône tronqué, un peu aplati inférieurement, échancré à sa base, laquelle est dirigée en arrière et en haut, et est beaucoup plus large que le sommet, qui est dirigé en avant et en bas. Chez quelques sujets elle est quadrilatère, aplatie de haut en bas, et échancrée supérieurement. Sa face supérieure est

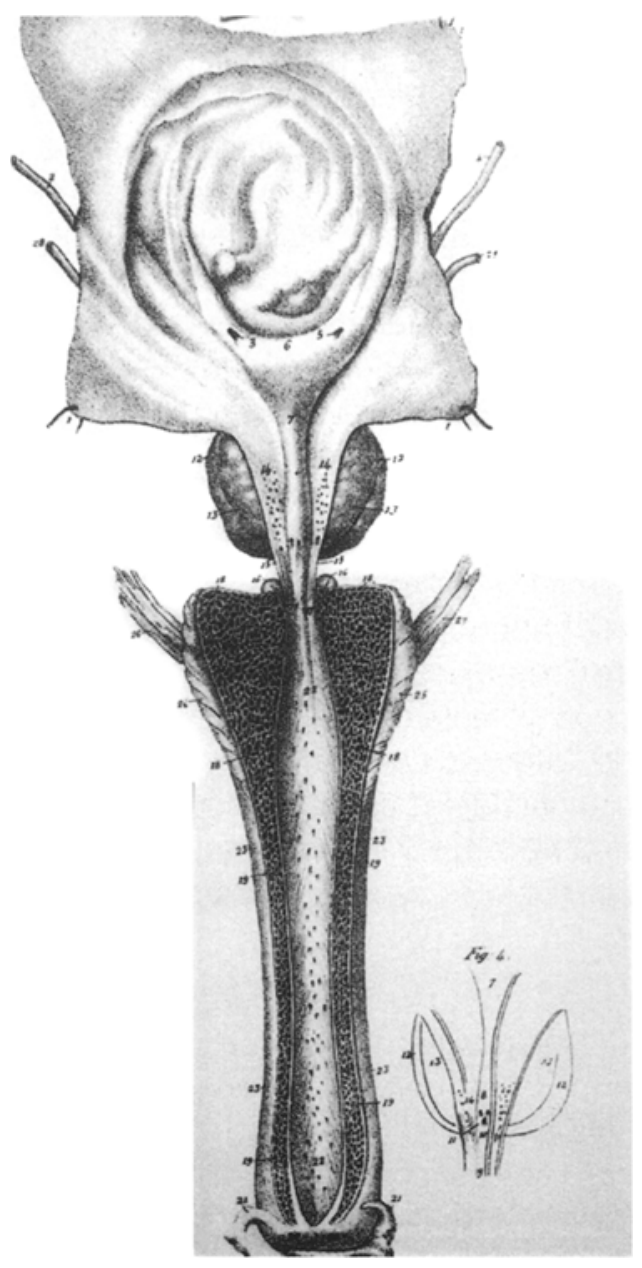

Figure 7 : La prostate et l'urètre. 
ordinairement recouverte dans toute son étendue par des fibres musculaires qui viennent du col de la vessie. Sa face inférieure offre un léger aplatissement, et adhère au rectum par du tissu cellulaire serré, dans lequel il ne s'amasse pas de graisse, et par l'aponévrose pelvienne. Ses parties latérales sont en rapport avec les muscles releveurs de l'anus. Sa base répond en haut au col de la vessie, et offre en cet endroit chez quelques sujets un tubercule ou bourrelet transversal, qu'Everard Home considère comme un troisième lobe, et qui fait saillir en haut le col de la vessie d'une manière plus ou moins prononcée. En bas, la base de la prostate est en contact avec les canaux déférents et les vésicules séminales. Son sommet paraît ordinairement comme tronqué derrière la portion membraneuse de l'urètre ; quelquefois, au contraire, il semble se prolonger sur elle. Sa circonférence présente inférieurement une excavation pour recevoir le rectum, dont la face antérieure est unie intimement à l'enveloppe fibreuse de la prostate par un tissu cellulaire très dense ; et supérieurement ce corps glanduleux est séparé de l'arcade du pubis par les muscles pubo-prostatiques, leurs ligaments, du tissu cellulaire et quelques branches de l'artère honteuse.

La prostate est traversée longitudinalement par l'urètre, qui est presque toujours plus voisin de sa face supérieure que de l'inférieure. Cependant cela n'est pas constant, car on a vu l'urètre traverser cet organe au-dessous de sa partie moyenne, ou même tout à fait inférieurement. Dans d'autres cas, au contraire, ainsi que l'a indiqué Amussat, la face supérieure de la prostate reçoit seulement l'urètre comme dans une espèce de rigole, la glande n'entourant ce conduit que dans ses trois quarts inférieurs ; le quart supérieur de l'anneau urétral est alors complété par des fibres musculaires qui paraissent être une continuation de la tunique charnue du col de la vessie, et qui, dans l'état ordinaire, recouvrent la face supérieure de la prostate. Suivant Velpeau, ces fibres musculaires formeraient à la prostate une enveloppe complète, mais très mince à la partie inférieure de l'organe. La prostate est aussi traversée d'arrière en avant par les canaux éjaculateurs, lesquels sont placés obliquement au-dessous de l'uretère. Elle est unie à ces divers canaux par du tissu cellulaire assez serré.

La prostate est entourée d'une espèce de capsule fibreuse ; cette capsule est formée par l'aponévrose que j'ai nommée pelvienne, et qu'on a appelée depuis recto-vésicale et aponévrose supérieure du périnée, au moment où elle abandonne la vessie et le rectum pour aller se fixer aux branches des pubis. Le tissu de la glande est d'un blanc grisâtre, très dense, et paraît composé de tissu cellulaire et d'un grand nombre de follicules remplis d'un liquide visqueux et blanchâtre. De ces follicules naissent dix à quinze conduits excréteurs qui viennent s'ouvrir dans l'urètre sur les côtés du verumontanum. Ils versent dans le canal une humeur visqueuse, destinée à le lubrifier, et à servir de véhicule à la liqueur spermatique pendant l'éjaculation.

Les glandes de Cowper sont deux petits groupes ovoïdes de follicules mucipares, rougeâtres, placés immédiatement au-devant de la prostate, derrière le bulbe de l'urètre et ses vaisseaux, et dont les canaux excréteurs s'ouvrent obliquement dans la portion membraneuse du canal.

II existe quelquefois aux environs du bulbe de l'urètre un ou plusieurs autres petits corps semblables, mais leur existence est loin d'être constante.

\section{Du Pénis et de l'urètre}

Le pénis ou la verge, (Figure 8) est un organe cylindroïde, allongé, érectile, situé au-devant et audessous de la symphyse des pubis et qui sert à l'excrétion de l'urine et du sperme. Dans l'état ordinaire la verge est molle, pendante au-devant des bourses ; pendant l'érection elle s'alionge, se redresse, et prend une forme triangulaire. Dans les deux cas, au reste, elle offre beaucoup de différences individuelles. Sa face supérieure a reçu le nom de dos de la verge ; sa face inférieure présente une saillie longitudinale formée par l'urètre ; les deux côtés de la verge sont arrondis; son extrémité postérieure ou sa racine est attachée au bassin ; son extrémité antérieure est libre, et présente le gland, le prépuce et l'orifice de l'urètre.

Le pénis (Figure 9) est formé par le corps caverneux, siège principal de l'érection, par l'urètre, canal excréteur commun de l'urine et du sperme, et par le gland qui termine ce canal. II est en outre recouvert par un prolongement des téguments, et soutenu par un ligament.

La peau du pénis (Figure 10) se continue avec celle du pubis et du scrotum ; elle est très mince, et unie au corps caverneux et à l'urètre par un tissu cellulaire lamelleux très lâche, qui lui permet de glisser sur ces parties, et qui communique immédiatement avec celui du scrotum. Ce tissu cellulaire ne contient jamais de graisse, mais il s'infiltre avec une grande facilité. Vers l'extrémité antérieure de l'organe, la peau se réfléchit sur elle-même, de devant en arrière, jusque derrière la base du gland en devenant plus rouge, plus mince encore, plus humide, plus sensible, et elle forme le prépuce, lequel est composé d'une lame extérieure continue avec la peau, et d'une lame interne qui est d'apparence muqueuse et se continue avec celle qui recouvre le gland. Ces deux lames sont très faiblement unies entre elles par une couche très mince du tissu cellulaire sous-cutané. 


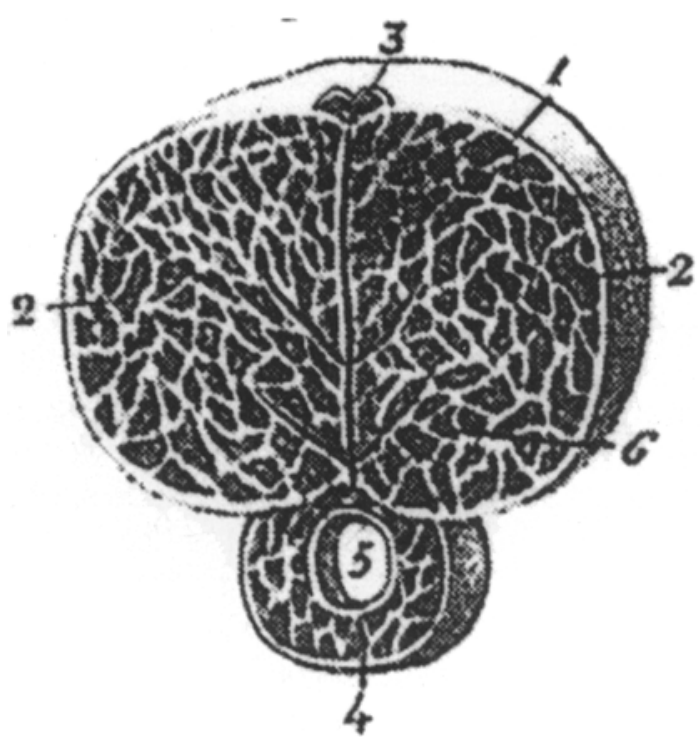

Figure 8 : Le pénis et l'urètre.

Le sommet du prépuce s'étend plus ou moins au-devant du gland, et présente une ouverture très variable dans ses dimensions ; sa base est fixée à une ligne ou deux derrière le gland, excepté à la partie inférieure, où il est uni à l'extrémité de l'urètre par un repli triangulaire qui $y$ adhère solidement, et auquel on donne le nom de frein du pénis. La peau du pénis contient un assez grand nombre de follicules sébacés, surtout à la partie inférieure de l'organe, où ils sont entourés de poils dirigés d'arrière en avant. Au-dessous du feuillet interne du prépuce, on trouve deux ou trois rangées de ces follicules qui fournissent une humeur onctueuse, épaisse, laquelle s'amasse en grande abondance dans certains cas de phimosis.

Le ligament suspenseur du pénis est un faisceau de forme triangulaire, aplati transversalement, qui s'étend de la partie antérieure et inférieure de la symphyse pubienne au corps caverneux de la racine du pénis. II est ordinairement fibro-celluleux, mais on y aperçoit quelquefois des fibres musculaires.

Les corps caverneux (Figure 10) qui constituent toute la partie supérieure et les parties latérales du pénis, sont formés par un tissu très compliqué de vaisseaux principalement veineux, entremêlés dans tous les sens, communiquant entre eux par de larges anastomoses, et entre lesquels se trouvent un grand nombre de filets nerveux et des prolongements d'une membrane fibreuse qui sert d'enveloppe aux corps caverneux eux-mêmes. Ces organes prennent naissance en arrière par deux racines, l'une droite, l'autre gauche, longues d'un pouce et demi à peu près, et fixées à la lèvre interne des

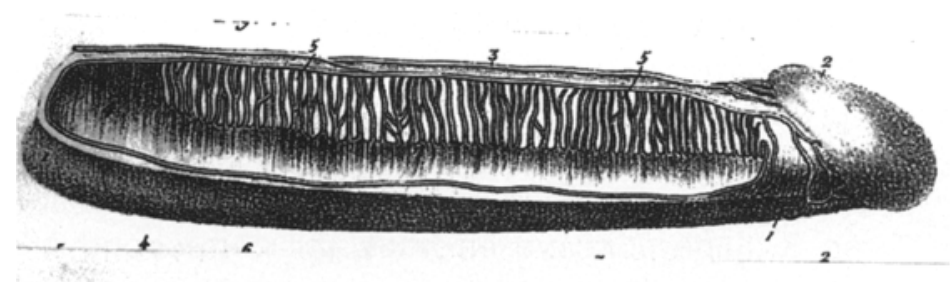

Figure 9 : Le pénis et l'urètre.

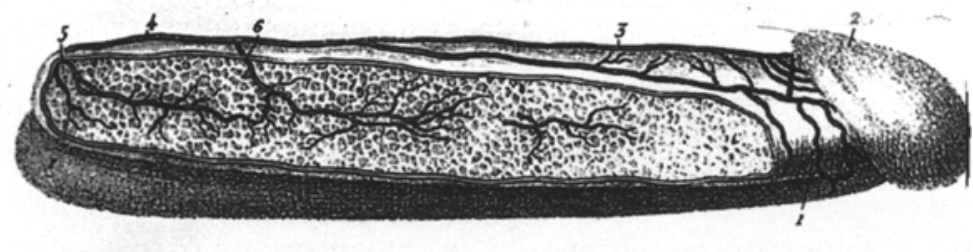

Figure 10 : Les corps caverneux.

branches ascendantes des ischions. Réunis au-devant de la symphyse pubienne, dans une enveloppe fibreuse commune, les corps caverneux sont partagés incomplètement en deux moitiés latérales par une cloison perpendiculaire (Figure 8). Cette cloison est un prolongement de la membrane fibreuse externe ; elle est étendue dans toute la hauteur des corps caverneux, et formée par des fibres qui produisent une cloison complète en arrière, et vont en s'amincissant et en diminuant en avant, où elles laissent entre elles des vides plus ou moins considérables. Ainsi réunis, les corps caverneux ont : une face supérieure qui reçoit postérieurement l'insertion du ligament suspenseur, et présente un sillon superficiel occupé par les vaisseaux dorsaux du pénis ; une face inférieure creusée d'un sillon plus profond et qui loge l'urètre; des faces latérales arrondies ; une extrémité antérieure représentant un cône tronqué et embrassé obliquement par le gland ; et une extrémité postérieure divisée en deux racines.

Les artères des corps caverneux viennent de la honteuse interne. Les principales sont les artères centrales, mais ils reçoivent des artères dorsales beaucoup de ramuscules qui percent la gaine. Les veines suivent le même trajet que les artères. Leurs nerfs proviennent des nerfs qui environnent les artères dorsales, avec lesquelles ils traversent la gaine fibreuse pour s'enfoncer dans le tissu caverneux.

L'urètre ou le canal excréteur de l'urine et du sperme chez l'homme (Figure 7), s'étend depuis le col de la vessie jusqu'à l'extrémité de la verge, en passant audessus de l'extrémité inférieure du rectum, au-dessous 
de la symphyse des pubis, et à la face inférieure des corps caverneux jusqu'au gland. L'urètre de l'homme a de sept pouces et demi à onze pouces de longueur. Dans l'état de flaccidité de la verge, et surtout quand le rectum est distendu, ce canal offre deux courbures en $S$, l'une concave en haut, qui correspond à la symphyse pubienne, et l'autre concave en bas, placée au-devant de cette articulation. Pendant l'érection, ou quand on relève le pénis contre l'abdomen, cette dernière courbure disparaît ; et Amussat a fait voir qu'on efface aussi presque complètement la courbure sous-pubienne lorsque, le rectum étant vide, on tire sur le pénis, de manière à l'incliner sur la face antérieure des pubis sous un angle d'environ quarante degrés. L'urètre n'a que trois portions bien distinctes, la portion prostatique, la portion membraneuse ou musculeuse, et la portion spongieuse.

La portion prostatique est longue de neuf à quinze lignes. Elle a la forme d'un cône, dont la base, tournée en arrière, a cinq lignes de diamètre chez un adulte et le sommet, dirigé en devant, trois lignes seulement. Elle est oblique d'arrière en avant et de bas en haut. On a vu plus haut comment la prostate se comporte à l'égard de l'urètre. Al'intérieur, ce canal présente sur sa paroi inférieure, et d'arrière en avant, une saillie transversale plus ou moins prononcée suivant les sujets, laquelle établit quelquefois une ligne de démarcation entre l'urètre et la vessie, et dépend soit du bourrelet transverse de la base de la prostate, soit de l'épaisseur du sphincter de la vessie, assez grande dans cet endroit. C'est à cette saillie que Amussat donne le nom de valvule pylorique de l'urètre. Au-devant d'elle on aperçoit une petite éminence fongueuse, qu'on appelle crête urétrale ou verumontanum.

La crête urétrale, placée longitudinalement sur la ligne médiane, divise la paroi inférieure de l'urètre en deux portions égales. Elle se termine environ à dix lignes en avant du col de la vessie, et forme en ce point une sorte de tubercule sur les côtés duquel viennent s'ouvrir les canaux éjaculateurs. Quelquefois ce tubercule est retiré sur lui-même, et présente une excavation. Ses parties latérales sont criblées par les orifices des conduits excréteurs de la prostate, lesquels représentent un $\mathrm{V}$ dont la pointe est dirigée en avant. De chaque côté du verumontanum on remarque un enfoncement plus ou moins considérable, suivant le volume du tubercule qui vient d'être décrit. La crête urétrale paraît n'être qu'un soulèvement de la membrane muqueuse, formé par les canaux éjaculateurs au moment où, se dégageant de la prostate, ils rampent entre cet organe et la tunique interne de l'urètre. On a vu naître de sa partie postérieure deux petits replis latéraux, concaves en avant, et qui avaient l'apparence de valvules. Dans les endroits où elle est percée par les conduits excréteurs de la prostate, la membrane muqueuse adhère fortement à cette glande ; c'est aussi à la partie inférieure du canal que les parois de cette portion de l'urètre sont plus minces ; leur épaisseur est plus grande dans la partie supérieure, où l'on distingue quelques fibres musculaires longitudinales et transversales quand la prostate n'entoure pas entièrement le conduit.

La portion membraneuse fait suite à la portion prostatique. En haut, elle est longue de huit à dix lignes, parce qu'elle se prolonge jusqu'à la portion spongieuse, et elle est placée directement sous le ligament triangulaire de la symphyse pubienne et sous la réunion des corps caverneux, dont elle est séparée, et auxquels elle adhère par du tissu cellulaire que traversent les artères et les veines dorsales de la verge. En bas, la portion membraneuse n'a guère plus de quatre lignes, parce que le sommet de la prostate et le bulbe de l'urètre semblent aller au-devant l'un de l'autre. Elle est en rapport, en arrière, avec la paroi antérieure du rectum, et en devant avec les glandes de Cowper et du tissu cellulaire d'apparence fibreuse qui la sépare du ligament périnéal ou aponévrose moyenne du périnée, et du muscle transverse.

La portion membraneuse est enveloppée par un prolongement de la gaine fibreuse de la prostate. Plus immédiatement, elle est embrassée par deux petits faisceaux musculaires lesquels s'attachent par un court tendon un peu au-dessus du bord inférieur de la symphyse des pubis, et aux piliers de l'aponévrose pelvienne. Quand ces muscles se contractent, ils rapprochent de l'arcade pubienne cette portion de l'urètre. Enfin chez les sujets jeunes et vigoureux on trouve, entre les muscles de Wilson et la membrane muqueuse, une couche plus ou moins épaisse de fibres longitudinales et circulaires, de nature musculaire, et qui paraissent n'être que la continuation des fibres musculaires du col de la vessie, après qu'elles ont enveloppé la portion prostatique de l'urètre. Quelquefois cette couche charnue est comme transformée en tissu jaunâtre. La portion membraneuse est cylindrique, légèrement renflée à son milieu, et plus rétrécie à son extrémité antérieure. Sa direction varie, comme celle de la portion prostatique, suivant que le rectum est vide ou rempli de matières.

La portion spongieuse est logée dans la rainure qu'on observe à la face inférieure des corps caverneux; elle est moins longue en haut qu'en bas. Dans ce dernier sens, en effet, le tissu dont elle est entourée, et qui lui donne son nom, se prolonge en arrière sous forme d'un renflement piriforme appelé bulbe de l'urètre. Le bulbe est séparé de l'extrémité inférieure du rectum par un 
intervalle qui varie de huit lignes à un pouce et il est uni à cet organe par du tissu cellulaire serré et par le muscle sphincter de l'anus. II est situé au-dessus du muscle bulbocaverneux et de la peau, en sorte que chez les personnes maigres on le sent facilement à l'extérieur. La portion de l'urètre qui correspond au bulbe est décrite par quelques anatomistes sous le nom de portion bulbeuse : nous l'avons comprise dans la portion membraneuse, parce qu'elle n'en est pas distincte, surtout supérieurement.

Le tissu spongieux de l'urètre décroît insensiblement de volume depuis le bulbe jusqu'au gland, qu'il forme par son épanouissement. Sa couche supérieure, ou celle qui correspond au corps caverneux, est peu épaisse ; l'inférieure n'est séparée de la peau que par le tissu cellulaire sous-cutané. Le tissu spongieux de l'urètre a la même organisation que les corps caverneux. La membrane fibreuse qui l'enveloppe est en contact en dedans avec la membrane muqueuse du canal urétral, et en dehors elle est unie à la gouttière des corps caverneux au moyen de filaments albuginés et par quelques vaisseaux. C'est sur le bulbe que se réunissent les deux feuillets dont elle se compose ; et Amussat a trouvé qu'en promenant le doigt d'avant en arrière sur la paroi inférieure de l'urètre, on sent à travers la membrane muqueuse une bride demi-circulaire qui, suivant lui, serait formée par la réunion de ces feuillets autour du tissu spongieux du bulbe. Quelques anatomistes pensent, au contraire, que cette bride, audessous et au niveau de laquelle se pratiquent quelquefois des fausses routes dans le cathétérisme, est formée par l'aponévrose périnéale.

Le diamètre de l'urètre dans sa portion spongieuse, diminue presque insensiblement d'arrière en avant jusque derrière le méat urinaire : dans cet endroit, le canal offre une dilatation plus ou moins marquée suivant les individus, mais toujours plus prononcée sur la paroi inférieure. On donne le nom de fosse naviculaire à cette dilatation, dont Amussat n'admet pas l'existence, et qui serait, selon cet anatomiste, une fausse apparence due à ce que le tissu du gland est moins mou et la muqueuse plus adhérente dans cet endroit. Enfin l'orifice extérieur de l'urètre est ordinairement fort étroit.

La membrane muqueuse de l'urètre est habituellement blanchâtre, et légèrement rosée près du méat urinaire. Elle présente, en haut et en bas, sur la ligne médiane, une ligne blanche plus ou moins distincte, que des anatomistes modernes regardent comme des sutures formées par la réunion des deux moitiés dont se compose l'urètre aux premiers temps de la vie intrautérine. On y aperçoit aussi des plis, dont les uns sont longitudinaux et bien marqués ; les autres sont moins prononcés, et ont une direction transversale, ou même une forme semilunaire, dont la concavité est tournée en avant. De plus la paroi inférieure de l'urètre est parsemée, surtout au niveau de la portion membraneuse, de lacunes muqueuses, dont les orifices sont dirigés en devant, et que l'on a désignées sous le nom de lacunes de Morgagni.

Les artères de l'urètre sont nombreuses, et viennent de la honteuse interne; les branches les plus grosses pénètrent dans le bulbe. Les veines suivent le trajet des artères. Ses vaisseaux lymphatiques se rendent dans les plexus inguinaux et hypogastriques. Ses nerfs viennent des nerfs honteux et petit sciatique.

Le gland forme l'extrémité du pénis, et a la forme d'un cône légèrement aplati. Son sommet, couvert par le prépuce ou libre suivant les individus, est percé par l'orifice de l'urètre. Sa base embrasse l'extrémité du corps caverneux, et lui est unie par des vaisseaux ; elle est circonscrite par un rebord saillant qu'on appelle la couronne du gland. Au-dessous de l'urètre, la couronne du gland est interrompue par un petit sillon qui s'étend jusqu'à l'orifice de ce conduit, et qui est rempli par le frein de la verge. Le gland est revêtu par une membrane muqueuse assez mince, garnie de follicules sébacés vers la couronne, et couverte d'un épiderme très fin. Son tissu intérieur est spongieux, érectile comme celui de la portion spongieuse de l'urètre, dont il n'est que la continuation et l'épanouissement.

\section{REFERENCES}

1. CLOQUET J.G. : Manuel d'anatomie descriptive du corps humain. Nouvelle édition en cinq volumes précédée d'un avis au lecteur sur la vie et l'œuvre de l'auteur par Liliane Pariente, $t$ V. Paris, Editions Louis Pariente, 1999 : 37-47.

2. HUGUET F. : Les professeurs de la Faculté de médecine de Paris. Dictionnaire biographique 1794-1939. Paris, Editions du CNRS, 1991: 115-117

3. RONSARD J. : Histoire de la chirurgie française au $19^{\mathrm{e}}$ siècle. Paris, Baillière, $1875:$ 178-180

4. VAPEREAU J. : Dictionnaire des contemporains. Paris, Doin, 1890.

Manuscrit reçu : juin 2006 ; accepté juin 2006. 


\section{ABSTRACT}

Andrological anatomy in the writings of Jules Germain Cloquet (1790-1883), a great anatomist and surgeon

\section{Georges ANDROUTSOS}

Cloquet was a great anatomist and surgeon. A large proportion of his writings, covering different fields of medicine, such as normal anatomy and physiology, pathology, natural history, and surgery, concerns andrological anatomy illustrating the astonishingly high level of Cloquet's knowledge in the field of andrology.

Key words: Cloquet, surgeon, anatomist, andrological anatomy 